\title{
Test-retest reproducibility of atomic force microscopy measurements of human trabecular meshwork stiffness
}

Larry Kagemann ${ }^{1-8}$, Joe Candiello2 , Gadi Wollstein ${ }^{1-4,6}$, Hiroshi Ishikawa ${ }^{1-4,6,10}$, Richard A. Bilonick ${ }^{1,3,11}$, Ian A. Sigal ${ }^{1-4}$, Christian Jonescu-Cuypers ${ }^{9}$, Prashant N. Kumta ${ }^{2,3,12,13}$, Joel S. Schuman ${ }^{1-4,6,10}$

${ }^{1}$ UPMC Eye Center, Eye and Ear Institute, Ophthalmology and Visual Science Research Center, Department of Ophthalmology, University of Pittsburgh School of Medicine, Pittsburgh, PA, USA; ${ }^{2}$ Department of Bioengineering, Swanson School of Engineering, University of Pittsburgh, Pittsburgh, PA, USA; ${ }^{3}$ McGowan Institute for Regenerative Medicine, University of Pittsburgh School of Medicine and University of Pittsburgh, Pittsburgh, PA, USA; ${ }^{4}$ The Louis J. Fox Center for Vision Restoration, University of Pittsburgh School of Medicine, Pittsburgh, PA, USA; ${ }^{5}$ US Food and Drug Administration, Silver Spring, MD, USA; ${ }^{6}$ NYU Langone Eye Center, NYU School of Medicine, New York University, New York, NY, USA; 'Department of Ophthalmology, School of Medicine, University of Maryland, Baltimore, MD, USA; ${ }^{8}$ Department of Surgery, Uniformed Services University of the Health Sciences, Bethesda, MD, USA; ${ }^{9}$ Department of Ophthalmology, Geneva University Hospitals, Geneva, Switzerland; ${ }^{10}$ Department of Biomedical Engineering, NYU Tandon School of Engineering, Brooklyn, NY, USA; ${ }^{11}$ Department of Biostatistics, Graduate School of Public Health, University of Pittsburgh, Pittsburgh, PA, USA; ${ }^{12}$ Department of Chemical and Petroleum Engineering, University of Pittsburgh, Pittsburgh, PA, USA; ${ }^{13}$ Department of Mechanical Engineering and Materials Science, University of Pittsburgh, Pittsburgh, PA, USA

\section{Abstract}

Purpose: The purpose of the present study was to quantify test-retest reproducibility of measurements of stiffness of the human trabecular meshwork (HTM) by atomic

Correspondence: Larry Kagemann, Ph.D., RAC, FARVO, US Food and Drug Administration, White Oak Building 66, Room 1252, 10903 New Hampshire Avenue, Silver Spring, MD 20993-0002, USA.

E-mail: Lawrence.Kagemann@fda.hhs.gov 
force microscopy (AFM).

Methods: Eleven $40 \mu \mathrm{m}$ radial limbal cryostat sections from a fresh human donor rim were mounted on charged slides and rehydrated at room temperature. Stiffness at four TM locations (anterior to posterior along Schlemm's canal) was measured by AFM. At each location, a $6 \times 6$ grid was sampled. Indentation points were evenly distributed over a $20 \mu \mathrm{m} \times 20 \mu \mathrm{m}$ area, with a rate of one load/unload cycle per second. Measurements were then repeated for calculation of test-retest variability. Results: The test-retest coefficients of variation for the four measurement locations (anterior to posterior) were $24.39,25.28,12.74$, and $14.26 \%$, respectively, with a notable drop in the two posterior locations compared to the anterior. The test-retest coefficient for the sections was $19.17 \%$. For the entire eye, the test-retest coefficient of variation for the measurement of the TM stiffness was $17.13 \%$. Young's moduli consistently decreased from anterior to posterior location.

Conclusions: Wide regional variation suggests that single value does little to fully describe the complex array of TM stiffness levels within the eye, and future studies of TM stiffness assessed by AFM should include multiple tissue samples from each eye, with documentation of the anterior-posterior location of each measurement.

\section{Introduction}

Elevated intraocular pressure (IOP) is the single most important risk factor in the diagnosis $^{1-3}$ and progression ${ }^{4,5}$ of glaucoma, and its reduction is the single clinical endpoint of treatment. ${ }^{6}$ Overby et al. recently demonstrated that gene expression in glaucoma is altered, resulting in elevated stiffening of the inner wall of Schlemm's canal, impeding formation of pores, leading to IOP elevation in glaucomatous eyes. To that end, there is increasing interest in the measurement of the stiffness of tissues in the proximal aqueous humor outflow pathway, including Schlemm's canal and the trabecular meshwork (TM).$^{7-13}$

A study by Last et al. suggests that TM stiffness is increased in glaucoma. ${ }^{7}$ In that study, stiffness (Young's modulus, E) as measured by atomic force microscopy (AFM) was found to vary by two orders of magnitude within individuals, and amongst subjects. AFM measurements of rat TM found that mean local stiffness changes by more than twenty-fold within individual eyes. ${ }^{11}$

We have successfully used AFM to quantify the stiffness of the basement membrane of the eye, but have not yet applied it to assess the measurement of TM stiffness. ${ }^{14,15}$ AFM is known to be a noisy measurement, ${ }^{16-22}$ although the test-retest reproducibility, i.e. the variation associated with repeated measurements of AFM assessment of TM stiffness, has not yet been quantified. The meaningful application of any technique to medical research first requires characterization of measurement error. The purpose of the present study was to quantify test-retest reproducibility of the human TM by AFM. 


\section{Methods}

The study was conducted in accordance with the tenets of the Declaration of Helsinki and the United States Health Insurance Portability and Accountability Act.

A right eye was obtained from a local eye bank (Center for Organ Recovery, and Education, Pittsburgh, PA, USA). The donor eye was from a 53-year-old female, and tested negative for HIV I/II plus O, HBCAb, HCV/HIV/HB/Nat, RPR. The eye was harvested and preserved by the eye bank at ten hours after death. Specifically, the eye was stored in Optisol (Chiron Ophthalmics, Irvine, CA, USA) at $-8^{\circ} \mathrm{C}$. Seven days after harvest, the cornea was removed for transplant and the rim was dissected and embedded in Tissue Tek Optimal Cutting Temperature Compound (Sakura Finetek USA Inc., Torrance, CA, USA) and stored at $-80^{\circ} \mathrm{C}$. Radial $40 \mu \mathrm{m}$ thick sections were cut on a cryostat (Leica CM3050 S cryostat, Leica Microsystems Inc., Buffalo Grove, IL, USA) and mounted on charged slides by an histotechnologist certified by the American Society for Clinical Pathology (ASCP).

TM stiffness was quantified by AFM standard accepted methods. ${ }^{14,23,24}$ Briefly, 11 tissue sections were reconstituted with phosphate-buffered saline (PBS) and allowed to rest at room temperature for 20 minutes. The slide was then placed in a MFP-3D-BIO Atomic Force Microscope (Asylum Research, Santa Barbara, CA, USA) mounted on an Olympus IX-71 fluorescence microscope (Olympus, Tokyo, Japan). Standard commercially available $100 \mu \mathrm{m}$ long Si3N4 cantilevers, with integrated pyramidal tips (Veeco, Inc, Santa Barbara, CA, USA) and a nominal spring constant (k) of $0.6 \mathrm{~N} / \mathrm{m}$ were used to indent the TM cells, calibrating the spring constant of each cantilever before each experiment. A $6 \times 6$ grid was sampled, with indentation points evenly distributed over a $20 \mu \mathrm{m} \times 20 \mu \mathrm{m}$ area. The measurement grid was applied in each of four areas (Fig. 1), with the AFM controlled by an automated process, at a rate of one load/unload cycle per second. The speed of the AFM tip indenting the tissue ranged from 2-10 $\mu \mathrm{m} / \mathrm{sec}$. The apparent Young's modulus of the tissue at each indentation point was calculated for each independent force-indentation curve using the Sneddon model. ${ }^{25}$ After completing measurements at each of the four areas (session A), the slide was removed and repositioned in the AFM microscope, and all measurements were repeated (session B) to quantify test-retest reproducibility.

Stiffness estimates were acquired at 4 locations in each of the tissue sections (Fig. 1). Each stiffness estimate was comprised of the average of 36 ( $6 \times 6$ grid, $20 \mu \mathrm{m}$ $x 20 \mu \mathrm{m}$ ) individual measurements. This methodology is a standard tissue-sampling technique, and offered as a default setting in our commercially available AFM unit. Test-retest coefficient of variability was calculated for each of the four measurement locations. The average of the four stiffness measurements was calculated to provide a mean stiffness for each section, and the test-retest coefficient of variability was calculated for the sections. Finally, the 11 tissue stiffness estimates were averaged to estimate TM stiffness for the eye. These values were calculated for both AFM runs (session $\mathrm{A}$ and session $\mathrm{B}$ ). 


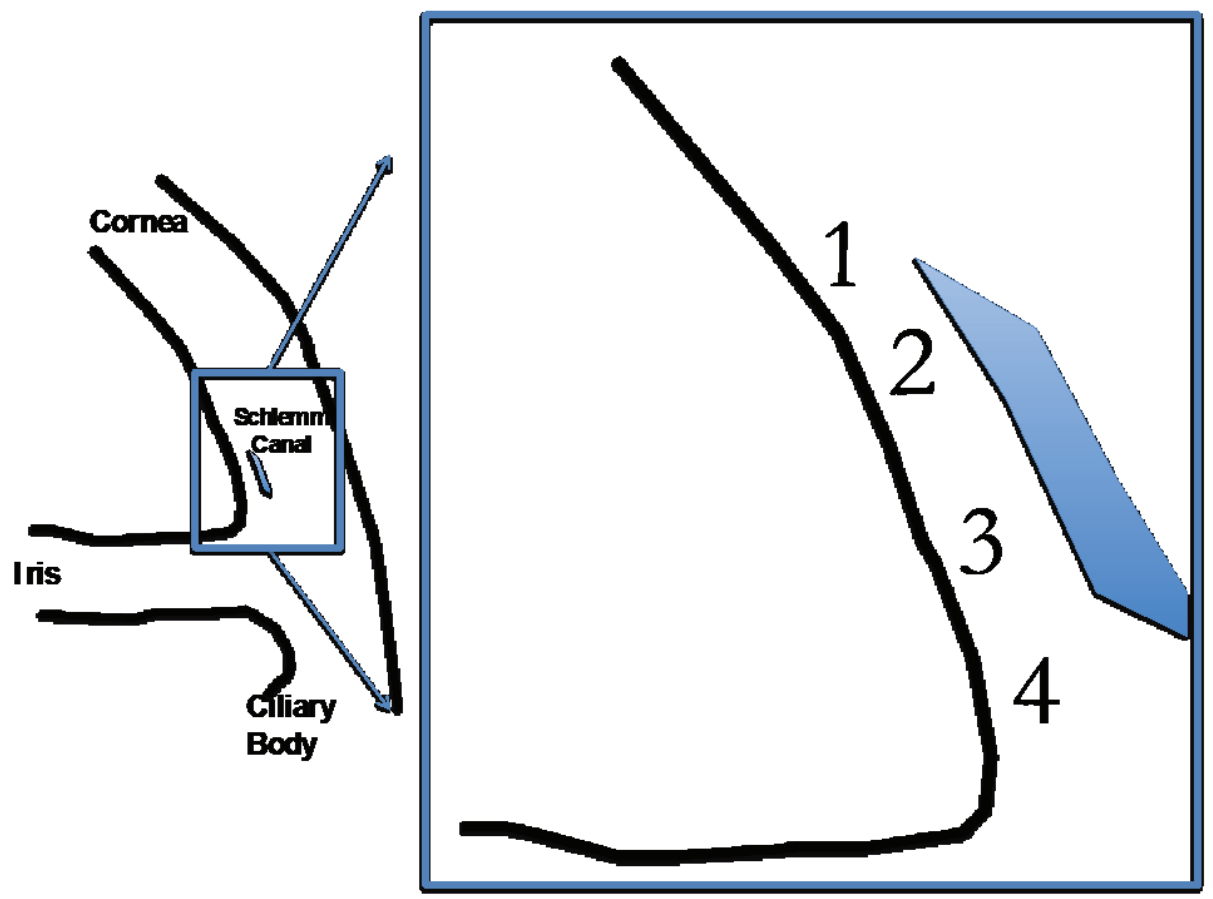

Fig. 1. The TM was measured at four locations from anterior to (1), adjacent to $(2,3)$, and posterior to (4) Schlemm's canal. Measurements at each location were obtained in sessions $A$ and $B$.

\section{Results}

The overall range of Young's moduli in sessions A and B were 8,376-242,733 and 5,574-130,352 Pa, respectively (Table 1). Moving from location 1 to 4, Young's moduli consistently decreased during both measurements $A$ and $B$. However, the location-to-location positional drop in Young's modulus was approximately $15 \mathrm{kPa}$ larger in session A compared to session B (13.4 kPa to $15.7 \mathrm{kPa}$, Fig. 2). The pattern of anterior to posterior TM softening (Fig. 2) was present throughout the series of 11 tissue slabs.

The test-retest coefficients of variation for the four measurement locations (anterior to posterior) were $24.39,25.28,12.74$, and $14.26 \%$, respectively, with a notable drop in the two posterior locations compared to the anterior. The test-retest coefficient for the sections was $19.17 \%$ (Table 1). For the entire eye, the test-retest coefficient of variation for the measurement of the TM stiffness was $17.13 \%$. 
Table 1. Mean and standard deviation stiffness measurements from sessions A and B for the 11 tissue sections, with coefficients of variation for each tissue section, the mean of the tissue sections, and the eye overall

\begin{tabular}{|l|l|l|l|}
\hline Section & Session A $(\mathbf{P a})$ & Session $\mathbf{B}(\mathbf{P a})$ & $\begin{array}{l}\text { Coefficient of } \\
\text { variation }\end{array}$ \\
\hline 1 & $15,721 \pm 9,434$ & $17,589 \pm 5,833$ & $8 \%$ \\
\hline 2 & $8,376 \pm 5,573$ & $5,574 \pm 2,684$ & $28 \%$ \\
\hline 3 & $31,649 \pm 5,485$ & $17,779 \pm 16,624$ & $40 \%$ \\
\hline 4 & $32,586 \pm 21,439$ & $38,652 \pm 17,250$ & $12 \%$ \\
\hline 5 & $25,424 \pm 7,478$ & $27,116 \pm 12,020$ & $5 \%$ \\
\hline 6 & $35,368 \pm 37,272$ & $38,687 \pm 17,250$ & $6 \%$ \\
\hline 7 & $38,977 \pm 38,589$ & $32,744 \pm 25,720$ & $12 \%$ \\
\hline 8 & $220,012 \pm 273,144$ & $130,352 \pm 108,487$ & $36 \%$ \\
\hline 9 & $49,262 \pm 58,909$ & $58,294 \pm 68,575$ & $12 \%$ \\
\hline 10 & $242,733 \pm 204,551$ & $126,484 \pm 89,613$ & $45 \%$ \\
\hline 11 & $83,345 \pm 39,141$ & $120,895 \pm 90,862$ & $26 \%$ \\
\hline Section mean & $71,223 \pm 63,720$ & $55,833 \pm 43,155$ & $\mathbf{1 7} \%$ \\
\hline Whole eye & & $63,528 \pm 53,438$ & $\mathbf{2 1} \%$ \\
\hline
\end{tabular}

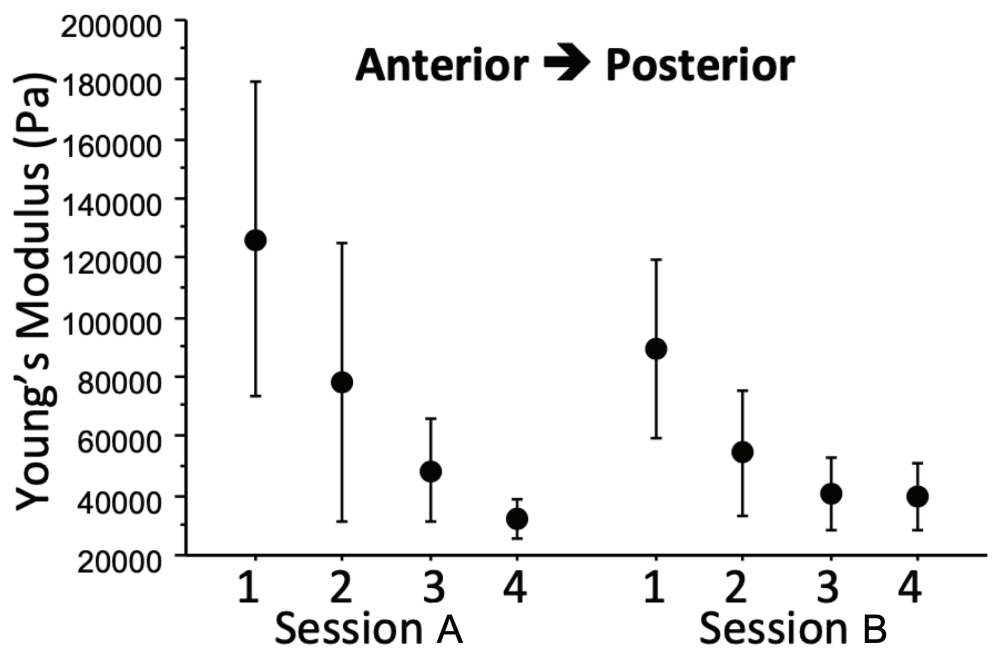

Fig. 2. Young's modulus presented with a trend of decrease with changing position from anterior to posterior locations in both sessions A and B; however, there were no statistically significant differences observed between the locations. 


\section{Discussion}

To date, published estimates of TM stiffness measured by AFM are comprised of single averages used to represent an entire eye., ${ }^{7,10}$ They present with variability greater than two orders of magnitude. The magnitude of the variability is unexplained, leaving the reader to speculate if its source is measurement error, true difference between sections, or true differences within the sections and between subjects. For example, the ranges of tissue stiffnesses (in $\mathrm{kPa}$ ) in glaucomatous eyes, by subject, were 1.4-329.7, 36.4-382.8, 1.7-565.3, 0.8-552.0, 23.2-126.6, 0.5-206.9, 2.0-243.0, $1.3-315.8,5.3-178.5$, and $1.5-142.5 .^{7}$ The present study provides the first systemic examination of the sources of variability in AFM measurements of TM stiffness. We found that AFM had a test-retest coefficient of variation $17 \%$ in an eye, and $19 \%$ for any individual tissue section. Further, we found that TM stiffness decreased with position from anterior to posterior.

A closer examination of the individual measurements suggests that the majority of test-retest discordance occurs in regions of high stiffness (Fig. 3). This may suggest that those anterior regions adjacent to the sclera (regions 1 and 2) contain a wider variety of small and large TM stiffness, unlike the posterior (regions 3 and 4), which present with relatively smaller levels of measurement variability (Fig. 2). Indeed, the large differences in those measurement locations with the highest stiffness values (Fig. 3) suggest a "hit or miss" phenomenon with respect to local regions of high stiffness, especially in the anterior-most location 1 . These data suggest that the
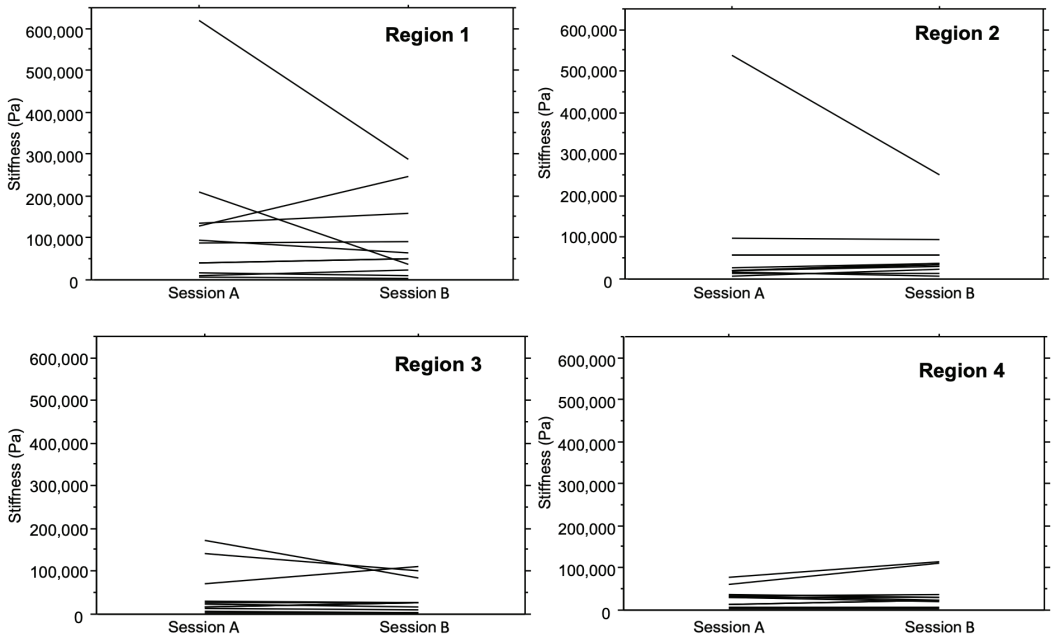

Fig. 3. Individual variation between the two sessions was small, with the exception of a small number of outliers in each region. Note that, in each case, large variability occurred in sections with high stiffness. 
most stable AFM measurements are to be found in the softer posterior-most region of the TM (Figs. 2 and 3). However, limiting assessment to the softest region of the $\mathrm{TM}$, by definition, biases the measurements to lower values.

The establishment of the level of measurement noise is necessary for power calculations in future studies. The present data also provides some insights into the reality of expected variability within individual tissue sections and individual eyes. The unexpected finding of a predictable pattern of regional variation within individual tissue slices, representing a predictable "normal" pattern of TM softening deep within the angle, may in itself serve as a biomarker for disease. However, in the present study, the tissue was completely relaxed, having been sectioned from a donor eye. It is possible that this pattern may be altered in living eyes due to the influence of muscle activity within the TM itself, as well as via influence of ciliary muscle activity transmitted to the TM via connecting tendons.

Regional differences throughout the eye suggest a need for a comprehensive assessment of TM stiffness. We do not yet fully understand the relationship between varied levels of TM stiffness and regional outflow. However, the presence of softer TM in the posterior location is consistent with histological observations in older eyes, specifically of pigment deposition in the TM adjacent to Schlemm's canal, but not anterior, marking posterior TM as the area of active flow. ${ }^{26}$ In this study, we found the posterior TM to have lower stiffness, also consistent with the hypothesis that regions of active outflow have lower TM stiffness.

The present study has several limitations. As the primary purpose of the study was to determine expected variability when measuring TM stiffness in a single eye, multiple sections from only one eye were used. Data from one eye are not generalizable to the population, and further studies are needed. However, the pattern observed in this human donor eye agrees with previous published findings in a rodent model. ${ }^{11}$ Surprisingly, despite measuring stiffness from an individual eye, the present study demonstrated that a wide range of stiffness values is present in an eye. Until we better understand the meaning of this range of values, an individual mean may not adequately quantify TM stiffness. The data suggest that tissue samples from numerous locations around the TM are needed, and within each sample, the anterior-posterior location should be documented. Further, in the present study, a pyramidal-tipped AFM probe was used. These tips are known to yield higher estimations of cell stiffness than spherical tips, but are valid for measurements in soft tissue. ${ }^{27}$ Previous studies have elected to use a probe with a spherical tip. ${ }^{7}$ The use of a pyramidal tip may yield stiffness estimates more affected by the TM cell cortex, but stiffness estimates in the present study ranged from a few to several hundred kilopascals; far larger than the small differences observed when comparing spherical and "sharp" AFM probe tips. ${ }^{28}$ There was good agreement between the first and second measurements of the tissue slices, suggesting that the performance of the pyramidal tipped AFM probe was reproducible (Fig 3). Finally, rehydrated tissue sections were used in the present study, as opposed to fresh 
whole-tissue sections. This is an accepted and previously published technique in the measurement of stiffness of the biological ophthalmic structures. ${ }^{23,24}$

AFM reveals local patterns of TM stiffness in the human eye. The relationship between this array of stiffness levels, morphology, and outflow has yet to be determined in either cadaveric flow models, or more importantly, in living healthy and glaucomatous eyes. Wide regional variation suggests that single value does little to fully describe the complex array of TM stiffness levels within the eye, and future studies of TM stiffness assessed by AFM should include multiple tissue samples from each eye, with documentation of the anterior-posterior location of each measurement.

\section{Acknowledgements}

Dr. Joel S. Schuman wishes to disclose that he received royalties for intellectual property licensed by the Massachusetts Institute of Technology and Massachusetts Eye and Ear Infirmary to Zeiss, Inc.

Dr. Prashant N. Kumta acknowledges the Edward R. Weidlein Chair Professorship and the Center for Complex Engineered Multi-functional Materials (CCEMM), Swanson School of Engineering, University of Pittsburgh for use of the AFM.

The contents of this publication are the sole responsibility of the author(s) and do not necessarily reflect the views, opinions, or policies of Uniformed Services University of the Health Sciences (USUHS), the US Food and Drug Administration, the Department of Defense (DoD), the Departments of the Army, Navy, or Air Force. Mention of trade names, commercial products, or organizations does not imply endorsement by the US Government.

This work was supported in part by National Institutes of Health (Bethesda, MD, USA) contracts R01-EY13178, and P30-EY08098, the Eye and Ear Foundation (Pittsburgh, PA, USA), and unrestricted grants from Research to Prevent Blindness (New York, NY, USA).

\section{References}

1. Leske MC, Connell AM, Schachat AP, Hyman L. The Barbados Eye Study. Prevalence of open angle glaucoma. Arch Ophthalmol. 1994;112:821-829.

2. Mitchell P, Smith W, Attebo K, Healey PR. Prevalence of open-angle glaucoma in Australia. The Blue Mountains Eye Study. Ophthalmology. 1996;103:1661-1669.

3. Sommer A, Tielsch JM. Risk factors for open-angle glaucoma: the Barbados Eye Study. Arch Ophthalmol. 1996;114:235. 
4. Drance S, Anderson DR, Schulzer M, Collaborative Normal-Tension Glaucoma Study G. Risk factors for progression of visual field abnormalities in normal-tension glaucoma. Am J Ophthalmol. 2001;131:699-708.

5. Leske MC, Hyman L, Hussein M, Heijl A, Bengtsson B. Comparison of glaucomatous progression between untreated patients with normal-tension glaucoma and patients with therapeutically reduced intraocular pressures. The effectiveness of intraocular pressure reduction in the treatment of normal-tension glaucoma. Am J Ophthalmol. 1999;127:625-626.

6. American Academy of Ophthalmology, Glaucoma Panel, Preferred Practice Pattern Guidelines. Primary Open-Angle Glaucoma. San Francisco CA, AAO. 2010.

7. Last JA, Pan T, Ding Y, et al. Elastic modulus determination of normal and glaucomatous human trabecular meshwork. Invest Ophthalmol Vis Sci. 2011;52:2147-2152.

8. Camras LJ, Stamer WD, Epstein D, Gonzalez P, Yuan F. Differential effects of trabecular meshwork stiffness on outflow facility in normal human and porcine eyes. Invest Ophthalmol Vis Sci. 2012;53:52425250.

9. Yu JG, Bao FJ, Feng YF, et al. Assessment of corneal biomechanical behavior under posterior and anterior pressure. J Refract Surg. 2013;29:64-70.

10. Camras LJ, Stamer WD, Epstein D, Gonzalez P, Yuan F. Circumferential tensile stiffness of glaucomatous trabecular meshwork. Invest Ophthalmol Vis Sci. 2014;55:814-823.

11. Huang J, Camras LJ, Yuan F. Mechanical analysis of rat trabecular meshwork. Soft Matter. 2015;11(14):2857-2865.

12. Morgan JT, Raghunathan VK, Chang YR, Murphy CJ, Russell P. The intrinsic stiffness of human trabecular meshwork cells increases with senescence. Oncotarget. 2015.

13. Morgan JT, Raghunathan VK, Chang YR, Murphy CJ, Russell P. Wnt inhibition induces persistent increases in intrinsic stiffness of human trabecular meshwork cells. Exp Eye Res. 2015;132:174-178.

14. Candiello J, Balasubramani M, Schreiber EM, et al. Biomechanical properties of native basement membranes. FEBS J. 2007;274:2897-2908.

15. Candiello J, Cole GJ, Halfter W. Age-dependent changes in the structure, composition and biophysical properties of a human basement membrane. Matrix Biol. 2010;29:402-410.

16. Ruozi B, Tosi G, Forni F, Fresta M, Vandelli MA. Atomic force microscopy and photon correlation spectroscopy: two techniques for rapid characterization of liposomes. Eur J Pharm Sci. 2005;25:81-89.

17. Trache A, Meininger GA. Atomic force-multi-optical imaging integrated microscope for monitoring molecular dynamics in live cells. J Biomed Optics. 2005;10:064023.

18. Tocha E, Schonherr H, Vancso GJ. Quantitative nanotribology by AFM: a novel universal calibration platform. Langmuir. 2006;22:2340-2350.

19. Tsuji T, Kobari K, Ide S, Yamanaka K. Suppression of spurious vibration of cantilever in atomic force microscopy by enhancement of bending rigidity of cantilever chip substrate. Rev Sci Instrum. 2007;78:103703.

20. Ying ZC, Reitsma MG, Gates RS. Direct measurement of cantilever spring constants and correction for cantilever irregularities using an instrumented indenter. Rev Sci Instrum. 2007;78:063708.

21. Akrami SM, Nakayachi H, Watanabe-Nakayama T, Asakawa H, Fukuma T. Significant improvements in stability and reproducibility of atomic-scale atomic force microscopy in liquid. Nanotechnology. 2014;25:455701. 
22. Demichelis A, Divieto C, Mortati L, Pavarelli S, Sassi G, Sassi MP. Toward the realization of reproducible Atomic Force Microscopy measurements of elastic modulus in biological samples. J Biomech. 2015;48:1099-1104.

23. Braunsmann C, Hammer CM, Rheinlaender J, Kruse FE, Schaffer TE, Schlotzer-Schrehardt U. Evaluation of lamina cribrosa and peripapillary sclera stiffness in pseudoexfoliation and normal eyes by atomic force microscopy. Invest Ophthalmol Vis Sci. 2012;53:2960-2967.

24. Marturano JE, Arena JD, Schiller ZA, Georgakoudi I, Kuo CK. Characterization of mechanical and biochemical properties of developing embryonic tendon. Proc Natl Acad Sci U S A. 2013;110:6370-6375.

25. Radmacher M, Fritz M, Hansma PK. Imaging soft samples with the atomic force microscope: gelatin in water and propanol. Biophys J. 1995;69:264-270.

26. Freddo T, Johnson M. Aqueous Humor Dynamics I: Measurement methods and animal studies. In: Civan MM (ed), The Eye's Aqueous Humor. Amsterdam, Boston, Heidelberg, London, New York, Oxford, Paris, San Diego, San Francisco, Singapore, Sydney, Tokyo: Elsevier; 2008.

27. Harris AR, Charras GT. Experimental validation of atomic force microscopy-based cell elasticity measurements. Nanotechnology. 2011;22:345102.

28. Vargas-Pinto R, Gong H, Vahabikashi A, Johnson M. The effect of the endothelial cell cortex on atomic force microscopy measurements. Biophys J. 2013;105:300-309. 